\title{
Simultaneous temperature estimation of multiple gratings using a multi-layer neural network
}

Djurhuus, Martin Søren Engmann; Schmauss, Bernhard; Clausen, Anders T.; Zibar, Darko

Published in:

IEEE Photonics Technology Letters

Link to article, DOI:

10.1109/LPT.2020.3019102

Publication date:

2020

Document Version

Peer reviewed version

Link back to DTU Orbit

Citation (APA):

Djurhuus, M. S. E., Schmauss, B., Clausen, A. T., \& Zibar, D. (2020). Simultaneous temperature estimation of multiple gratings using a multi-layer neural network. IEEE Photonics Technology Letters, 32(19), 1257 - 1260. https://doi.org/10.1109/LPT.2020.3019102

\section{General rights}

Copyright and moral rights for the publications made accessible in the public portal are retained by the authors and/or other copyright owners and it is a condition of accessing publications that users recognise and abide by the legal requirements associated with these rights.

- Users may download and print one copy of any publication from the public portal for the purpose of private study or research.

- You may not further distribute the material or use it for any profit-making activity or commercial gain

- You may freely distribute the URL identifying the publication in the public portal 


\title{
Simultaneous temperature estimation of multiple gratings using a multi-layer neural network
}

\author{
Martin S.E. Djurhuus, Bernhard Schmauss, Anders T. Clausen and Darko Zibar
}

\begin{abstract}
This paper introduces a method to do simultaneous temperature estimations of multiple gratings in an FBG array. The method involves training a multi-layer neural network using simulated training data. The network is then used to estimate the temperature changes of multiple gratings simultaneously using only the experimentally obtained spectrum of the FBG array. The versatility of the method is seen from the results of three different setups. That is the broadband lightsource with OSA, the broadband lightsource with spectrometer, and incoherent optical frequency domain reflectometry. The method can estimate the temperature changes with high accuracy and low root mean squared error (RMSE) for the setups under consideration. Finally, the method is shown to be capable of simultaneous estimation of temperature changes for 19 FBGs using the BLS setup with a maximum absolute error of $6.64 \mathrm{~K}$ and an RMSE of $1.69 \mathrm{~K}$ in only $1.74 \mathrm{~ms} / \mathrm{FBG}$.
\end{abstract}

Index Terms-Optical fiber sensors, Bragg gratings, Machine learning, Neural network, FBG arrays

\section{INTRODUCTION}

Fibre Bragg gratings (FBG) are commonly used in the measurement of physical parameters such as strain, vibration, pressure, and temperature [1] [2]. This is due to their advantages such as electrical interference immunity, remote sensing suitability, small size, and multiplexing capabilities [3]. The FBG structure is a wavelength specific reflector and is used as a sensor because the centre wavelength shifts if the physical parameters change [3]. The spectral shift caused by temperature changes is given by:

$$
\Delta \lambda_{B}=\lambda_{B}(\alpha+\eta) \Delta T
$$

where $\lambda_{B}, \Delta T, \alpha$, and $\eta$ is the centre wavelength, the temperature change, and two material constants [3].

Typically the detection scheme for FBG monitoring consists of measuring the FBG reflection spectrum. This can be done with multiple FBGs multiplexed in the same fibre using wavelength division multiplexing (WDM) and/or time division multiplexing. The conventional data processing algorithms find the centre wavelength of the FBG spectrum and compares it to a reference to find the shift and calculates the temperature using Eq. 1 for a particular FBG [4]. These algorithms include centroid detection, Gaussian fitting, and correlation [4]. These methods are fast due to their simple calculations but the accuracy of the methods can be compromised due to resolution issues or non-symmetric reflection spectrum. The Gaussian fit takes $1.75 \mathrm{~ms}$ with a root mean squared error (RMSE) $2{ }^{\circ} \mathrm{C}$ per $\mathrm{FBG}$ for a simulation. Evolutional algorithms such as the dynamic multiswarm particle swarm optimizer [5] and the genetic algorithm [6] have also been attempted. They have the advantage of accuracy and the ability to detect spectrally

M. Djurhuus and B. Schmauss are with the Institute of Microwaves and Photonics, Friedrich-Alexander University Erlangen-Nürnberg.

M. Djurhuus, A. Clausen and D. Zibar are with the Department of Photonic Engineering, Technical University of Denmark. overlapping FBGs at the cost of speed. Recent papers have attempted to implement this process using machine learning techniques such as online sequential extreme learning machine [7], backpropagation neural network [8], and radial basis function neural network [9]. These implementations of the methods are accurate but have high processing time.

An issue with all of these algorithms is that because each FBG is treated separately, the calculation of temperatures for all the FBGs can have long processing times. This is the case even if the individual processing time is short as some arrays can have thousands of FBGs [10]. This makes real-time monitoring of large FBG arrays difficult.

A multi-peak detection method is described in [11]. But this method focusses on accuracy instead of speed, is only tested on four FBGs, and for optical spectrum analyzer (OSA) measurements. The method's error is given as a maximum absolute error (MAE) of 0.03-0.07 ${ }^{\circ} \mathrm{C}$. Another method described in [12] focusses on solving the problem of spectrally overlapping FBGs with good accuracy (RMSE of $0.0095^{\circ} \mathrm{C}$ ) but with a processing time of $0.132 \mathrm{~s} / \mathrm{FBG}$. This method is also only tested on four FBGs and for OSA measurements.

This paper employs a machine learning approach based on multi-layer neural networks for simultaneous estimation of the temperature of multiple FBGs. The method is tested on different setups such as a broadband lightsource with an OSA, a broadband lightsource with a spectrometer, and incoherent optical frequency domain reflectometry (iOFDR). The performance is investigated in terms of MAE, RMSE, processing time per FBG, and number of FBGs. This paper differs from our previous paper [13] in four aspects. Firstly the model is trained from simulated data, Secondly, this model uses a neural network. Thirdly, this paper looks at multigrating estimation. Finally, an iOFDR method [14] has been added to the measurements. These differences make the new method more versatile, robust, and fast at the cost of accuracy.

\section{EXPERIMENTAL SETUP AND DATA COLLECTION}

The proposed method introduces a multi-layer neural network to process an FBG array by estimating the temperatures of multiple FBGs simultaneously. The concept is to teach the network the relationship between the multi-FBG spectra and a vector of each FBG's temperature using simulated data. Then the model is using experimental data to estimate the temperatures $T_{n k}$ based on the combined spectra $R_{k}$ of the FBGs as shown in Fig. 1.

Three different setups are used to investigate the performance of the method. Setup 1 is a broadband lightsource with an OSA abbreviated BLO and is seen in the upper part of Fig. 2. This setup uses an EDFA as a broadband source for an FBG array. The reflected spectrum is recorded using an 


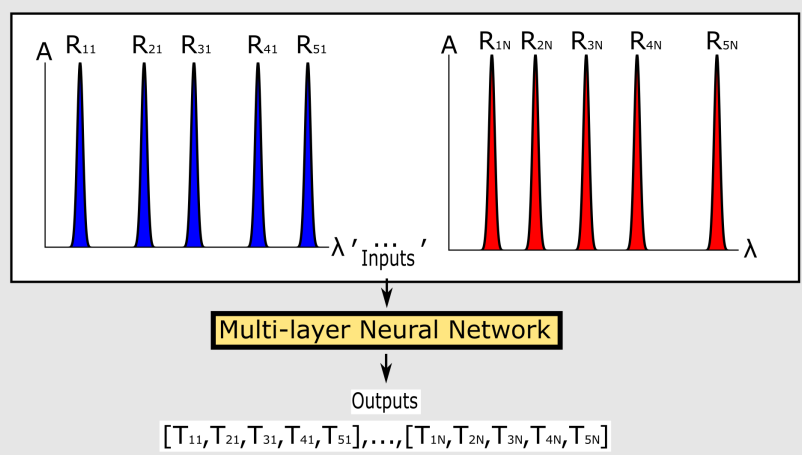

Fig. 1: The concept behind the proposed method of mapping the temperatures $T_{n k}$ from the reflection spectra $R_{n k}$ where $n$ represents a specific FBG and $k$ represents a certain temperature configuration. An example of the simultaneous estimation of temperatures of multiple FBGs using their reflection spectra is shown here using five FBGs for simplicity.

OSA. The OSA settings are a $0.05 \mathrm{~nm}$ resolution and a 1510$1590 \mathrm{~nm}$ span. The temperature of the FBG array is controlled using a climate chamber. Setup 2 is a broadband lightsource with a spectrometer abbreviated BLS and can also be seen in the upper part of Fig. 2. Here a spectrometer is recording the spectrum with a wavelength range of 1509.2 to 1595.2 in 512 points. Setup 3 is an iOFDR setup shown in the lower part of Fig. 2. Here Peltier modules control the temperature. The light source and detector are a tunable laser source (TLS) and a photodetector (PD). A Vector Network Analyser (VNA) with a built-in signal generator is connected to the PD and a MachZehnder modulator (MZM). The setup modulates the output of the TLS with an RF signal that changes in frequency from $1 \mathrm{MHz}$ to $2.5 \mathrm{GHz}$ in steps of $1 \mathrm{MHz}$ using the MZM. The reflected light from the FBGs is detected by the PD and the VNA records the transfer function of the system. By tuning the wavelength of the TLS the transfer function at different optical wavelengths is recorded.

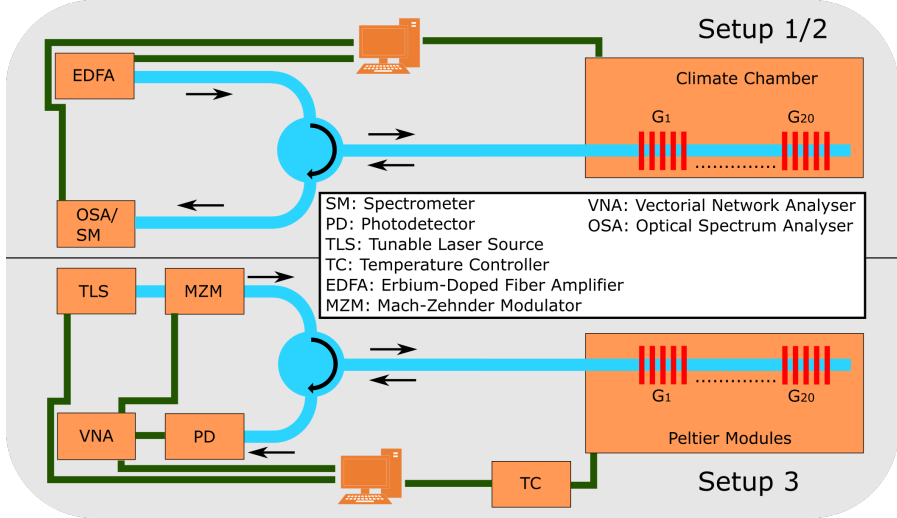

Fig. 2: Sketch of the setups used to acquire the experimental data. The sketch is split into two with two setups BLO and BLS in the upper part and the iOFDR in the bottom.

The BLO setup is included in the paper as a performance baseline. The OSA is often used to measure FBG spectra and it is therefore of interest to prove that the method works for these measurements. Furthermore, the OSA spectrum has a lot of points included which means that more spectrum information is available compared to more sparsely sampled methods. The BLS setup is included since the spectrometer makes the fastest measurements, which makes it the most interesting for realtime measurements, and is the cheapest detector. The iOFDR setup is included as this setup makes it possible to increase the number of measurable FBGs outside the WDM limitations by adding a spacial dimension to the measurements. This is also the slowest method as it records an extra dimension.

The FBG array used has 20 FBGs. The FBGs are positioned from $1512 \mathrm{~nm}$ to $1588 \mathrm{~nm}$ with a separation of $4 \mathrm{~nm}$, all with a spectral FWHM width of $0.2 \mathrm{~nm}$ and a reflection of $20 \%$.

For the BLO and BLS setups the temperature changes from -20 to $80{ }^{\circ} \mathrm{C}$ in steps of $1{ }^{\circ} \mathrm{C}$. For the iOFDR setup the temperature changes from 10 to $80^{\circ} \mathrm{C}$ in steps of $1{ }^{\circ} \mathrm{C}$. This is done due to equipment constraints. For each temperatures, the spectra and temperature are recorded. For the iOFDR setup, the transfer functions for wavelengths from 1527 to $1573 \mathrm{~nm}$ in steps of $0.1 \mathrm{~nm}$ are recorded instead of the spectra.

\section{DATA GENERATION AND PROCESSING}

In this paper, the training data are simulated and the test data are experimental. The simulated data are generated by replicating the shift of the FBG spectra associated with temperature changes as shown by Eq. 1. The process is as follows:

1) The temperature changes are chosen. This is done according to the data generation method in [15] by drawing temperature changes from a uniform distribution between $-60 \mathrm{~K}$ and $60 \mathrm{~K}$. These are organized into $K=10^{6}$ vectors of size $N$ equal to the number of modelled FBGs.

2) A base spectrum is chosen. This consists of a spectrum containing the peaks of $N$ FBGs for a known temperature. A experimental reference spectrum is found to be a good base spectrum. For the BLO results, the base spectrum is downsampled by a factor of 10 to prevent memory issues.

3) The shift is simulated using a Fourier transform, multiplying a phase and using an inverse transform. The phase is $\phi=\exp \left(-\left(i 2 \pi c \Delta \lambda_{B} t\right) / \lambda_{0}^{2}\right)$ where $c$ is the speed of light, $t$ is the time axis after the Fourier transform, $\lambda_{0}$ is the spectrum centre wavelength and $\Delta \lambda_{B}$ is the shift calculated from Eq. 1 where appropriate material constants are found.

4) Finally, to prevent over-fitting, white Gaussian noise is added to the resulting spectrum $R_{k}$ of size $M$.

The resulting data set consists of the spectra and their corresponding temperature changes denoted $D=$ $\left\{R_{1 k}, \ldots, R_{M k}, T_{1 k}, \ldots, T_{N k} \mid k=1, \ldots, K\right\}$. The spectra are $K$ vectors of size $M$. The $N$ FBG peaks are shifted separately and normalised to be between 0 and 1 . The temperatures are $K$ vectors of size $N$ of temperature changes.

The experimental data processing starts after the data acquisition. The data consist of temperatures and the corresponding spectra. Due to equipment limitations, all of the FBGs have the same temperature. As the iOFDR method does not measure spectra but transfer functions a conversion is needed. The iOFDR data consist of one transfer function for each step of the TLS per temperature measured. Therefore the transfer functions are Fourier transformed to give the impulse response 
of the system for each optical wavelength measured. The resulting spacial dimension of the impulse response is used to select an area of interest. This area is summed to eliminate the spacial dimension and create a spectrum equivalent corresponding to the measured temperature. The BLO spectra are again downsampled by a factor of 10 . From here the data processing is the same for all setups. It proceeds as follows:

1) The spectra are limited to the $N$ modelled FBGs. This is done to mirror the base spectrum used for the training data.

2) The spectra are normalized to remove the effects of the amplitude by scaling the spectra to run between 0 and 1 . This is done for each FBG by splitting the spectra into 4 $\mathrm{nm}$ pieces centred around an FBG peak. The pieces are then scaled and put back into the spectrum. This results in a spectrum where all FBG peaks have the peak value 1 .

3) The temperatures are converted into temperature changes using the base spectrum temperature and put in appropriate $N$ sized temperature vectors.

The resulting test and training data are given to a multi-layer back-propagation neural network model. The model consists of three hidden layers with 64 nodes in each and the activation functions tanh, sigmoid and tanh. Convergence was achieved after 50 epochs with a training RMSE of 0.79 and a validation RMSE of 1.15. The computer used for data processing has an intel Xeon 3.6 GHz CPU and 16 GB RAM.

\section{RESULTS AND DisCUSSION}

In Fig. 3a, 3b and 3c the estimated temperature changes are plotted for the measured data. The temperature changes of five FBGs are simultaneously estimated for each setup under consideration. The estimated temperature changes for each of the FBGs are plotted against the measured temperature changes. The ideal estimation where the measured and estimated temperature changes are the same is shown as a dashed line. The RMSE of the test set is in the plot title and the correlation coefficients $r$ for each FBG are in the legend.

From Fig. 3a it is seen that the simultaneous estimation of temperature changes for the five FBGs does not significantly deviate from the ideal line. This is also seen from the correlation coefficients between $0.9991-0.9993$. The MAE is $3.46 \mathrm{~K}$ and the RMSE is $1.77 \mathrm{~K}$. The estimations all deviate together which could be calibration errors where the FBG temperature and the recorded temperature differ. The processing time for one spectrum with five FBGs is $0.1306 \mathrm{~s}$ with downsampling and $0.042 \mathrm{~s}$ without.

From the plots in Fig. $3 b$ it is seen that the estimated temperature changes are closer to the ideal line than for the BLO case. This is backed up by the correlation coefficients between 0.9997-0.9998. The RMSE of $1.3 \mathrm{~K}$ is also lower than the BLO case. The MAE is $3.76 \mathrm{~K}$. The better performance compared to the BLO case is due to the lower number of input dimensions caused by a more sparsely sampled spectrum. This means that less data are needed to train the model. Finally, the deviations around $-50 \mathrm{~K}$ seen in estimations are the same for all the FBGs. This could again indicate calibration errors. The processing time for one spectrum with five FBGs is $0.031 \mathrm{~s}$.

Fig. 3c shows the estimated temperature changes for the iOFDR setup. Here the correlation coefficients between 0.9992-0.9998 are slightly worse than the BLS case. The MAE is $3.38 \mathrm{~K}$. From the RMSE of $1.03 \mathrm{~K}$ it is seen that the performance is even better than the BLS case. In this case, the source is coherent making the noise of the iOFDR data lower than the BLS data. The deviation from the ideal increases below $-20 \mathrm{~K}$ because the Peltier modules have problems cooling the fibre creating a difference between the measured temperature and the temperature of the FBGs. This is also the reason that the correlation coefficients are worse than the BLS case. The processing time for one spectrum with five FBGs is $3.705 \mathrm{~s}$ due to the Fourier transforms used.

The results presented in these three figures show that the use of the proposed method to simultaneously estimate temperature changes of multiple FBGs from their spectrum using experimental data is possible with good accuracy. The BLS results are seen to be the fastest from the processing time. This combined with the short measurement time of the spectrometer shows a possible use in real-time measurement applications. If similar results are possible when more FBGs are modelled, it would seriously bring down the processing time per FBG.

To show the performance for an increased number of

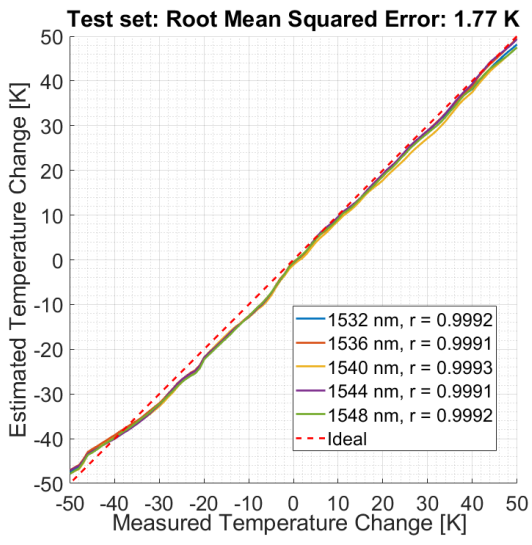

(a) BLO setup results.

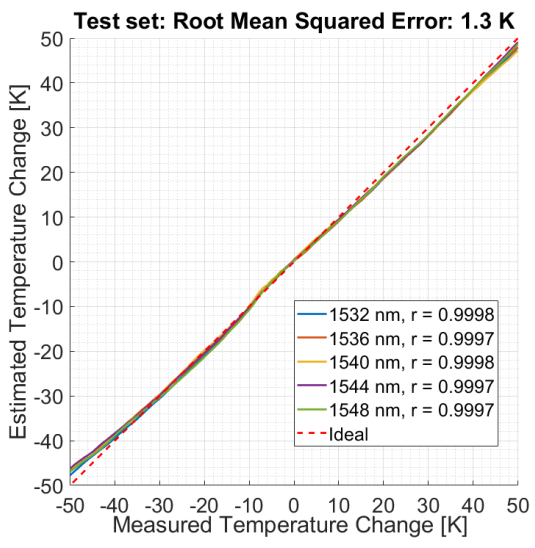

(b) BLS setup results.

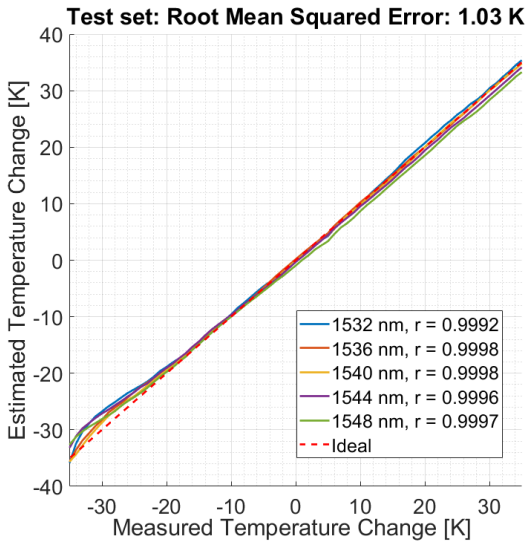

(c) iOFDR setup results.

Fig. 3: Plot of the estimated temperature changes using simulated training and experimental test data. The dashed line is the ideal result while the others are the estimated results for five FBGs measured experimentally. 
gratings, 19 FBGs from the spectrometer data are used. 19 FBGs are used since the 20th FBG shifts outside the recorded spectrum for certain temperatures. The spectrometer data are used since it is the fastest measurement method. Furthermore, the iOFDR data have 12 FBGs recorded due to source limitations and the BLO data have memory issues if 13 or more FBGs are modelled, which means that similar plots using these data could not be generated. The simultaneously estimated temperature changes of 19 FBGs are shown in Fig. 4. The estimated temperature changes for each of the 19 FBGs are plotted separately against the measured temperature changes.

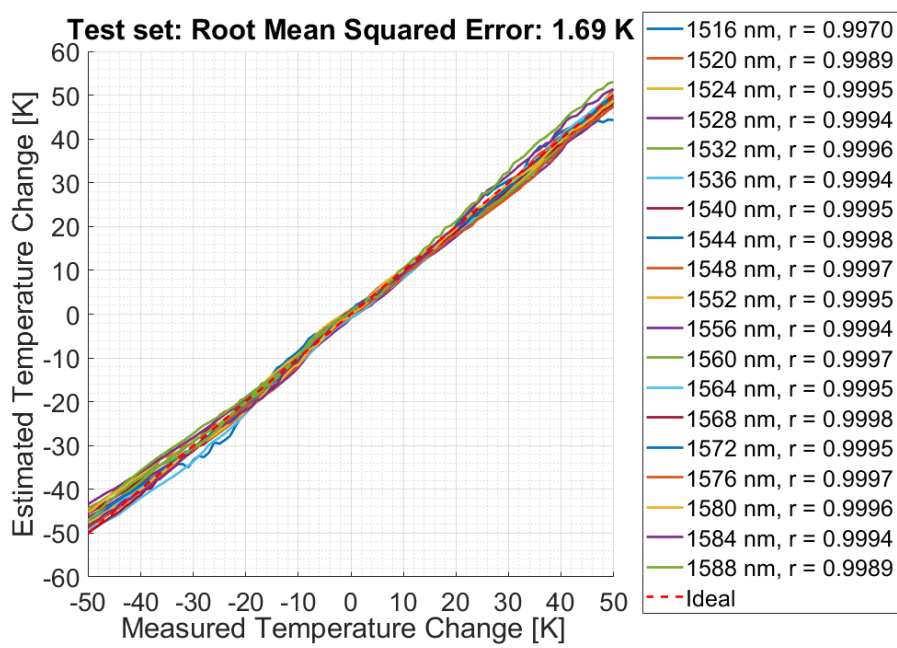

Fig. 4: Plot of the estimated temperature changes using simulated training and experimental test data. The dashed line is the ideal result while the others are the estimated results for 19 FBGs measured experimentally using a spectrometer.

The $r$ values in Fig. 4 show that the estimated temperature changes for most of the 19 FBGs are quite close to the ideal line. Compared to the experimental five FBG results, the result is worse than the BLS and iOFDR but better than the BLO case. This is not surprising due to the curse of dimensionality [16]. As such with the number of training points kept constant a decrease in the performance is to be expected. The performance compared to the BLO case is better because the BLO results have an even lower training points to input dimension ratio. This performance is seen from both the low RMSE of $1.69 \mathrm{~K}$ and the high $\mathrm{r}$ values, which are lower and higher than the BLO case. The deviations increase with distance to $0 \mathrm{~K}$ could be due to a wrong estimate of the slope in Eq. 1. The MAE is $6.64 \mathrm{~K}$. From the r values FBG 1516, 1520 and $1588 \mathrm{~nm}$ have a lower $\mathrm{r}$ value than the other FBGs. These FBGs peaks were closer to the noise floor due to source limitation. As such the results are not that surprising. Due to these issues in further works a wider source could be useful. The processing time for one spectrum with 19 FBGs is 0.033 $\mathrm{s}$ which is $1.74 \mathrm{~ms} / \mathrm{FBG}$.

From these results, it is seen that the method can be used for practical applications for at least 19 FBGs. As described earlier real-time measurement applications should be possible due to the short processing time of $1.74 \mathrm{~ms} / \mathrm{FBG}$ as long as an error of $6.64 \mathrm{~K}$ can be tolerated. Compared to the conventional method the Gaussian fit, the RMSE is 0.31
$\mathrm{K}$ lower and the processing time is the same [4]. Finally, as equipment limitation prevented the FBGs from having different temperatures, a numerical analysis using test data with 19 gratings and arbitrary temperatures generated with the same process as the training data was carried out and gave a RMSE of $1.32 \mathrm{~K}$. This shows that the method should be applicable even when the FBG temperatures are different from each other.

\section{CONCLUSION}

This paper has proposed an experimentally tested method for simultaneous estimation of temperature changes of multiple FBGs. The versatility of the method has been shown from the results of three different experimental setups. These results show that the proposed method can simultaneously estimate the measured temperature changes of five FBGs with RMSE's of $1.77,1.3$ and $1.03 \mathrm{~K}$. Finally, the method is shown to be capable of simultaneous estimation of the temperature changes for 19 FBGs using the BLS setup with an MAE of $6.64 \mathrm{~K}$ and an RMSE of $1.69 \mathrm{~K}$ in only $1.74 \mathrm{~ms} / \mathrm{FBG}$.

\section{REFERENCES}

[1] A. Kersey, M. Davis, H. Patrick, M. LeBlanc, K. Koo, C. Askins, M. Putnam, and E. Friebele, "Fiber grating sensors," Journal of Lightwave Technology, vol. 15, no. 8, pp. 1442-1463, 1997.

[2] U. Senkans, J. Braunfelds, I. Lyashuk, J. Porins, S. Spolitis, and V. Bobrovs, "Based Sensor Networks and Their Coexistence with Fiber Optical Transmission Systems," 2019.

[3] M. M. Werneck, R. C. S. B. Allil, B. A. Ribeiro, and F. V. B. de Nazaré, "A Guide to Fiber Bragg Grating Sensors," in Current Trends in Shortand Long-period Fiber Gratings. InTech, may 2013.

[4] D. Tosi, "Review and Analysis of Peak Tracking Techniques for Fiber Bragg Grating Sensors," Sensors, vol. 17, no. 10, pp. 1-35, 2017.

[5] J. Liang, P. Suganthan, C. Chan, and V. Huang, "Wavelength detection in FBG sensor network using tree search DMS-PSO," IEEE Photonics Technology Letters, vol. 18, no. 12, pp. 1305-1307, jun 2006.

[6] C. Shi, C. Chan, W. Jin, Y. Liao, Y. Zhou, and M. Demokan, "Improving the performance of a FBG sensor network using a genetic algorithm," Sensors and Actuators A: Physical, vol. 107, no. 1, pp. 57-61, oct 2003.

[7] Q. Shang and W. Qin, "Fiber bragg grating dynamic calibration based on online sequential extreme learning machine," Sensors (Switzerland), vol. 20, no. 7, apr 2020.

[8] Y. An, X. Wang, Z. Qu, T. Liao, and Z. Nan, "Fiber Bragg grating temperature calibration based on BP neural network," Optik, vol. 172, pp. 753-759, nov 2018.

[9] Y. An, X. Wang, Z. Qu, T. Liao, L. Wu, and Z. Nan, "Stable temperature calibration method of fiber Bragg grating based on radial basis function neural network," Optical Engineering, vol. 58, no. 09, p. 1, sep 2019.

[10] Z. Luo, H. Wen, H. Guo, and M. Yang, "A time- and wavelength-division multiplexing sensor network with ultra-weak fiber Bragg gratings," Optics Express, vol. 21, no. 19, p. 22799, sep 2013.

[11] Y. Chen, K. Yang, and H. L. Liu, "Self-adaptive multi-peak detection algorithm for FBG sensing signal," IEEE Sensors Journal, vol. 16, no. 8, pp. 2658-2665, apr 2016.

[12] Y. C. Manie, J. W. Li, P. C. Peng, R. K. Shiu, Y. Y. Chen, and Y. T. Hsu, "Using a machine learning algorithm integrated with data denoising techniques to optimize the multipoint sensor network," Sensors (Switzerland), vol. 20, no. 4, feb 2020.

[13] M. S. Djurhuus, S. Werzinger, B. Schmauss, A. T. Clausen, and D. Zibar, "Machine learning assisted fiber bragg grating-based temperature sensing," IEEE Photonics Technology Letters, vol. 31, no. 12, pp. 939-942, jun 2019.

[14] S. Werzinger, M. Gottinger, S. Gussner, S. Bergdolt, R. Engelbrecht, and B. Schmauss, "Model-based compressed sensing of fiber Bragg grating arrays in the frequency domain," 25th Optical Fiber Sensors Conference (OFS), pp. 1-4, apr 2017.

[15] D. Zibar, A. M. Rosa Brusin, U. C. De Moura, F. Da Ros, V. Curri, and A. Carena, "Inverse System Design Using Machine Learning: The Raman Amplifier Case," Journal of Lightwave Technology, vol. 38, no. 4, pp. 736-753, feb 2020

[16] C. M. Bishop, Pattern Recognition and Machine Learning, 2013. 